\title{
Synthesis and characterization of poly(St-co-DVB)/mont- morillonite nanocomposites by suspension polymerization
}

\author{
Jiaming Ji*, KeSheng Cao, Hua Tang, ChangSheng $L i^{\star}$
}

"School of Materials Science and Engineering, Jiangsu University, Zhenjiang 212013, P.R. China; e-mail: jijiaming@126.com, lichangsheng@ujs.edu.cn; tel. +86 5118879 0268; fax: +8651188790268.

(Received: 12 June, 2009; published: 22 January, 2012)

\begin{abstract}
Poly(styrene-co-divinylbenzene)/organic montmorillonite (OMMT) nanocomposites were prepared by suspension polymerization. OMMT was prepared by ion exchange method between sodium montmorillonite (SMMT) and cetyltrimethyl ammonium bromide (CTAB) in an aqueous solution. The character and structure of OMMT was revealed by XRD and FT-IR. The microstructure of polymer/OMMT nanocomposites was characterized by XRD, TEM and SEM. The thermal stability of polymer/OMMT nanocomposites was studied by TG and DTG. Also the effect of the amount of OMMT and divinylbenzene (DVB) on the thermal stability of polymer/OMMT nanocomposites has been discussed in this paper. It was observed that the crosslinked polymer/clay nanocomposites exhibited better thermal stability than pure polystyrene and polystyrene/clay nanocomposites under the experimental conditions.

Key Words: suspension polymerization; montmorillonite; styrene; divinylbenzene; nanocomposite.
\end{abstract}

\section{Introduction}

Polymer layered silicates nanocomposites have been extensively used in transportation, construction, and electronics products. Significantly, they exhibit superior properties in comparison to their corresponding virgin polymers, such as electrical properties, mechanical property, thermal stability, barrier property, biodegradation, photodegradation as well as flame retardance behavior [1-8]. Recently, the polymer clay nanocomposites have attracted a deepening interest. Polymer clay nanocomposites are characterized by the size of the silicate layers with a nano-scaled dispersion in matrix, which have relatively large surface areas and interact strongly with polymer matrix. Therefore, the nanocomposites possessing superior properties attribute to the unique intercalation or exfoliation characteristics [9-10].

Montmorillonite (MMT) is a classic layered silicate for nanocomposite applications. Its structure is built from two tetrahedral silicate $\left(\mathrm{SiO}_{4}\right)$ layers and an octahedral layer of $\mathrm{AlO}_{6}$. Natural montmorillonite cannot be well dispersed in polymer matrix on account of its hydrophilic nature. However tetrahedral and octahedral cations can be replaced by lower valence cations, such as $\mathrm{Na}^{+}, \mathrm{Mg}^{2+}$, and $\mathrm{Al}^{3+}$, which can be exchanged with quaternary salts for preparing organic montmorillonite [11]. The role of the OMMT with quaternary salts is to increase the interlayer spacing, reduce aggregation between the montmorillonite sheets, and improve the compatibility between the clay and the polymer matrix [12].

Several useful polymer/montmorillonite nanocomposites have been prepared, such as 
polyamide-6/montmorillonite [13], poly(methyl methacrylate)/montmorillonite nanocomposites [14] and polypropylene/ montmorillonite nanocomposites [15]. Many researchers have studied the thermal stability of polymer/montmorillonite nanocomposites, for example, Wei-Ta Yang et al. [16] prepared polystyrene/clay nanocomposites by suspension and emulsion polymerization. The intercalated nanocomposites with the maximum d001 spacing of $3.3 \mathrm{~nm}$ and the maximum decomposed temperature of $42{ }^{\circ} \mathrm{C}$ were obtained. The decomposed temperature of composites with montmorillonite content of $5 \mathrm{wt} \%$ enhanced $18{ }^{\circ} \mathrm{C}$ compared with pure polystyrene. The polymer/montmorillonite nanocomposites exhibit drastic improvements in thermal stability, generally attributed to the uniform dispersion of the clay silicate layers in the polymer matrix. Some researchers attempted to prepare crosslinked polymer/clay nanocomposites for increasing the thermal stability. Seok-Kee Yoon et al. [17] prepared poly(St-co-DVB)/montmorillonite nanocomposites by $\mathrm{Y}$-ray irradiation. They found that the crosslinked copolymer/montmorillonite nanocomposites had better thermal stability than polystyrene/montmorillonite nanocomposites. This is because the addition of divinylbenzene can form crosslinked composites. However, compared with $y$-ray radiant polymerization, suspension polymerization with water as a solvent is a very popular technique for preparing polymer/clay nanocomposites, and gives little pollution.

In this paper, poly(styrene-co-divinylbenzene)/clay nanocomposites were prepared by suspension polymerization. The polymer/clay nanocomposites were characterized by XRD, FT-IR, TG, SEM and TEM. The results confirmed that the crosslinked nanocomposites were obtained by suspension polymerization and the crosslinked polymer/clay nanocomposites had better thermal stability than pure polystyrene or polystyrene/clay composites.

\section{Results and discussion}

\section{Organic montmorillonite}

Fig.1 shows the FT-IR patterns of SMMT (a) and OMMT (b) prepared by ion exchange method between SMMT and CTAB in an aqueous solution.

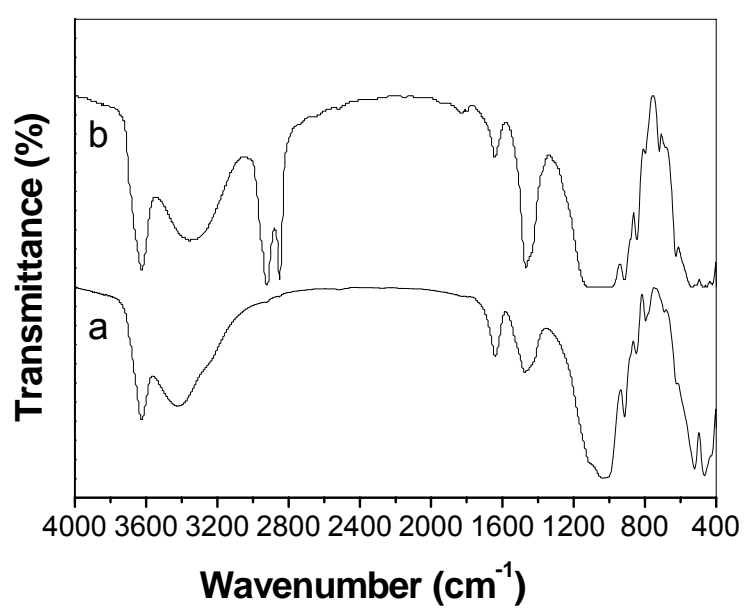

Fig.1. FT-IR spectras of SMMT (a) and OMMT (b). 
In Fig.1 (a), the characteristic peaks of SMMT were clearly seen in spectrums at $426 \mathrm{~cm}^{-1}, 523 \mathrm{~cm}^{-1}$ and $1035 \mathrm{~cm}^{-1}$ corresponding to Si-O-Si deformation, Si-O-Al and Si-O stretching and at $1641 \mathrm{~cm}^{-1}$ the stretching bands of O-H. Fig.1 (b) is the FT-IR patterns of OMMT and its characteristic peaks are shown at $2919 \mathrm{~cm}^{-1}$ and $2851 \mathrm{~cm}^{-1}$ corresponding to the $\mathrm{C}-\mathrm{H}_{2}$ asymmetric and symmetric stretching respectively [18]. These results indicated that CTAB had been successfully adsorbed on the interlayer of SMMT.

Fig. 2 shows the XRD patterns of SMMT (a) and OMMT (b) prepared by ion exchange method between SMMT and CTAB in an aqueous solution. In Fig.2 (a), the strong diffraction peak of SMMT appeared at $2 \theta=5.961^{\circ}$, which corresponded to its interlayer spacing $\mathrm{d} 001=1.481 \mathrm{~nm}$ based on Bragg equation $\lambda=2 \mathrm{~d} \sin \theta$.

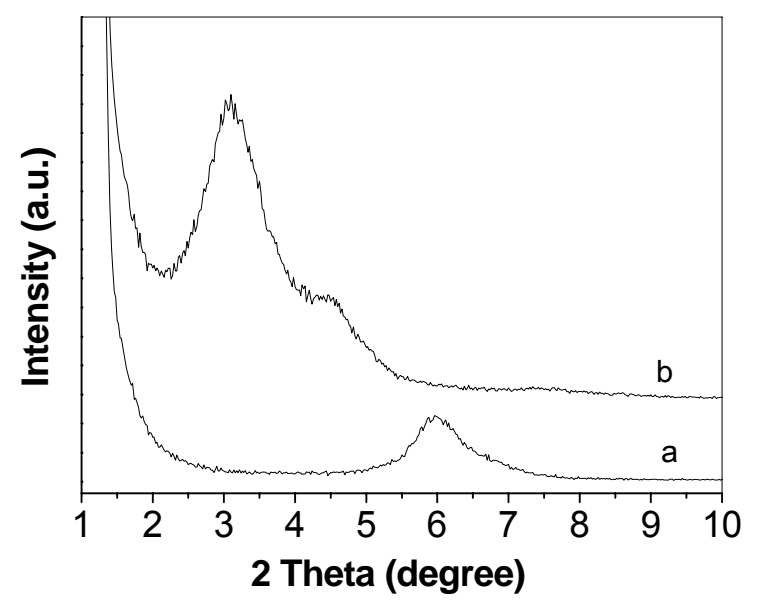

Fig. 2. X-ray diffraction patterns of SMMT (a) and OMMT (b).

It can be seen from Fig.2 (b), the strong diffraction peak of OMMT was $2 \theta=3.600^{\circ}$ and its interlayer spacing d001=2.452 nm. Compared with SMMT, the X-ray diffraction peak of OMMT shifted toward to lower angle and the interlayer spacing of OMMT increased. These results further confirmed that CTAB has been successfully inserted into the interlayer of SMMT. The increase of the interlayer spacing of OMMT is probably owing to the long-chains of CTAB inserted into the interlayer of SMMT [12]. It was well-known that the surface energy of OMMT was lower than SMMT, and then OMMT was more compatible with organic monomers [16]. Additionally, a weak diffraction peak of OMMT was also observed at $2 \theta=4.586^{\circ}(\mathrm{d}=1.925 \mathrm{~nm})$, which could probably correlate to the 002 plane of the montmorillonite layers [19].

\section{FT-IR and XRD of polymer/clay nanocomposites}

Fig. 3 shows the FT-IR patterns of SMMT (a), PS (b), St-co-DVB (c), PS/OMMT nanocomposite (d) and St-co-DVB/OMMT nanocomposite (e). Fig.3 (a) shows the characteristic peaks of MMT. It can be seen from Fig. 3 (b, c), the absorption peaks of benzene ring at $3056 \mathrm{~cm}^{-1}, 3026 \mathrm{~cm}^{-1}$, corresponding to $\mathrm{C}=\mathrm{C}$ stretching on benzene ring, and $1601 \mathrm{~cm}^{-1}, 1492 \mathrm{~cm}^{-1}, 1449 \mathrm{~cm}^{-1}$, corresponding to C-C stretching on benzene ring. Fig.3 (d, e) are the FTIR spectrum of PS/OMMT nanocomposite and St-co-DVB/OMMT nanocomposite. It can be seen from the figures that all the nanocomposites show the characteristic peaks of montmorillonite and benzene ring. These results confirmed that the OMMT has been dispersed in polymer via suspension polymerization. 


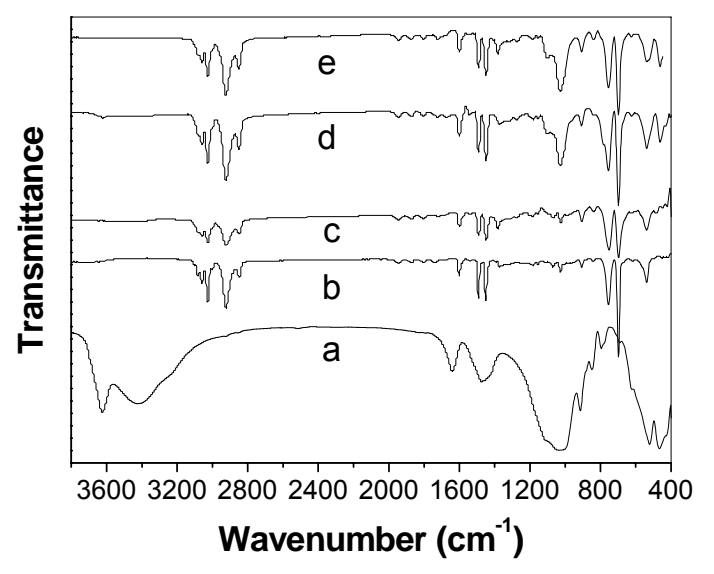

Fig. 3. FT-IR spectra of SMMT (a), PS (b), St-co-DVB (c), St/OMMT nanocomposites (d) and St-co-DVB/OMMT nanocomposites (e).

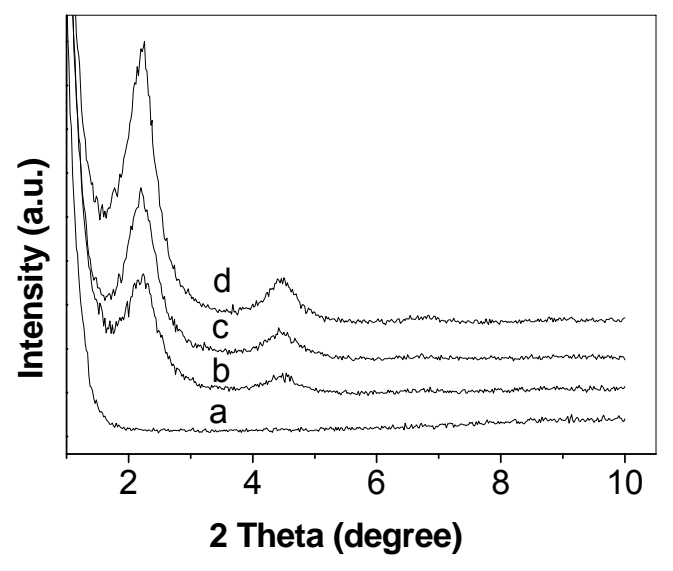

Fig. 4. XRD patterns of polymer/OMMT nanocomposites with DVB content of $2 \mathrm{wt} \%$ and various contents of OMMT: 0 (a), 3 (b), 5 (c) and 8 wt\% (d).

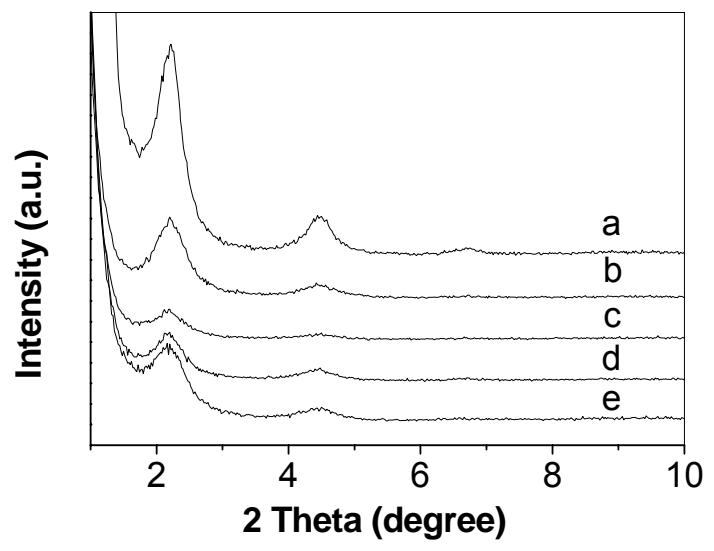

Fig. 5. XRD patterns of polymer/OMMT nanocomposites with OMMT content of $5 \mathrm{wt} \%$ and various contents of DVB: 0 (a), 2 (b), 4 (c), 6 (d) and $10 \mathrm{wt} \%$ (e). 
Fig. 4 shows the XRD patterns of polymer/OMMT nanocomposites prepared with DVB content of 2 wt \% and various OMMT contents of 0 (a), 3 (b), 5 (c) and 8 wt\% (d) by suspension polymerization. Fig.4 (a) shows the X-ray diffraction curves of the St-co-DVB copolymer without OMMT, which did not exhibit any diffraction peak, but when the different content of the OMMT (3, 5 and 8 wt\%) dispersed in polymer, all the nanocomposites exhibited intensified diffraction peak at $2.240^{\circ}, 2.201^{\circ}$ and $2.259^{\circ}$ corresponding to their interlayer spacing of $3.941 \mathrm{~nm}, 4.011 \mathrm{~nm}$ and $3.908 \mathrm{~nm}$, respectively. Compared to the interlayer spacing of OMMT(2.452 nm), the interlayer spacing of polymer/OMMT nanocomposites (about $4.0 \mathrm{~nm}$ ) had increased, which mean that the intercalation polymerization has happened. But with the addition of various OMMT content (3, 5 and $8 \mathrm{wt} \%$ ), the interlayer spacing of polymer/OMMT nanocomposites did not alter distinctly, indicating that the interlayer spacing of the polymer/OMMT nanocomposite was hardly affected by the amount of the OMMT. Hence, it can be concluded that perfect exfoliation of the clay layer structure of the OMMT in polymer did not occur [20]. Additionally, all of polymer/OMMT nanocomposites exhibited a weak diffraction peak at about $2 \theta=4.400^{\circ}$, which could probably correlate to the 002 plane of the montmorillonite layers dispersed in polymer [19].

Fig. 5 shows the XRD patterns of polymer/OMMT nanocomposites prepared with OMMT content of $5 \mathrm{wt} \%$ and various DVB contents of $0(\mathrm{a}), 2(\mathrm{~b}), 4(\mathrm{c}), 6(\mathrm{~d})$ and $10 \mathrm{wt} \%(\mathrm{e})$ by suspension polymerization. With the addition of various DVB content $(2,4,6$, and $10 \mathrm{wt} \%)$, all the nanocomposites exhibited intensified diffraction peak at $2.220^{\circ}, 2.201^{\circ}, 2.199^{\circ}, 2.260^{\circ}$ and $2.160^{\circ}$, corresponding to their interlayer spacing of $3.976 \mathrm{~nm}, 4.011 \mathrm{~nm}, 4.013 \mathrm{~nm}, 3.906 \mathrm{~nm}$ and $4.086 \mathrm{~nm}$. This also indicated that the interlayer spacing of the polymer/OMMT nanocomposite was hardly affected by the amount of the DVB.

\section{Thermal stability of polymer/clay nanocomposites}

Fig. 6 shows the TG(a) and DTG(b) curves of polymer/OMMT nanocomposites with DVB content of $2 \mathrm{wt} \%$ and various OMMT contents of $0(1), 3(2), 5(3)$, and $8 w t \%(4)$ prepared by suspension polymerization. Seen from Fig.6 (a), it can be observed that in $50 \%$ weight loss, the decomposition temperature of polymer without OMMT was $397{ }^{\circ} \mathrm{C}$, whereas the decomposition temperature of polymer/OMMT with the OMMT content of 3,5 , and 8 wt $\%$ was $410{ }^{\circ} \mathrm{C}, 420{ }^{\circ} \mathrm{C}$ and $414{ }^{\circ} \mathrm{C}$, respectively. In Fig.6 (b), it clearly exhibited that the maximum mass loss temperature of polymer without OMMT was $404{ }^{\circ} \mathrm{C}$, whereas the maximum mass loss temperature of polymer/OMMT with the OMMT content of 3,5 , and 8 wt $\%$ was $417{ }^{\circ} \mathrm{C}, 424{ }^{\circ} \mathrm{C}$ and $415^{\circ} \mathrm{C}$, respectively. The TG and DTG curves shown in Fig. 6 can confirm that the polymer/OMMT nanocomposite with the OMMT content of $5 \mathrm{wt} \%$ exhibited the best thermal stability compared to the others. This could probably be attributed to the fact that the appropriate OMMT has better compatibility with polymer and well-dispersed montmorillonite layers can handicap heat transfer between areas and also strengthen the polymer chain to avoid heat degradation [21].

Fig. 7 shows the TG(a) and DTG(b) curves of polymer/OMMT nanocomposites with OMMT content of $5 \mathrm{wt} \%$ and various DVB content of $0(1), 2(2), 4(3), 6(4)$ and $10 \mathrm{wt} \%(5)$ prepared by suspension polymerization. In Fig.7 (a), it can be observed that in $50 \%$ weight loss, the decomposition temperature of polymer/OMMT nanocomposite without DVB was $408{ }^{\circ} \mathrm{C}$, whereas the decomposition temperature of polymer/OMMT nanocomposites with the DVB content of 2, 4, 6 and $10 \mathrm{wt} \%$ was 419 
${ }^{\circ} \mathrm{C}, 421{ }^{\circ} \mathrm{C}, 422{ }^{\circ} \mathrm{C}$ and $428{ }^{\circ} \mathrm{C}$, respectively. In Fig.7 (b), it clearly exhibited that the maximum mass loss temperature of polymer/OMMT nanocomposite without DVB was $416{ }^{\circ} \mathrm{C}$, whereas the maximum mass loss temperature of polymer/OMMT nanocomposites with the DVB content of 2, 4, 6 and $10 \mathrm{wt} \%$ was $423{ }^{\circ} \mathrm{C}, 424{ }^{\circ} \mathrm{C}, 426$ ${ }^{\circ} \mathrm{C}$ and $437{ }^{\circ} \mathrm{C}$, respectively. The TG and DTG curves shown in Fig. 7 can make sure that the polymer/OMMT nanocomposite exhibited better thermal stability as the amount of DVB increased. This could probably be that DVB and styrene can form crosslinked copolymer which limits the movement of polymer chains and enhance the thermal stability of polymer/OMMT nanocomposites.

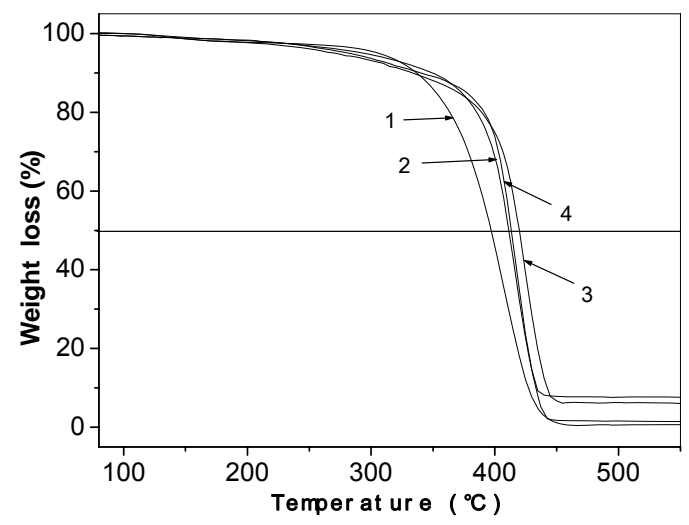

(a)

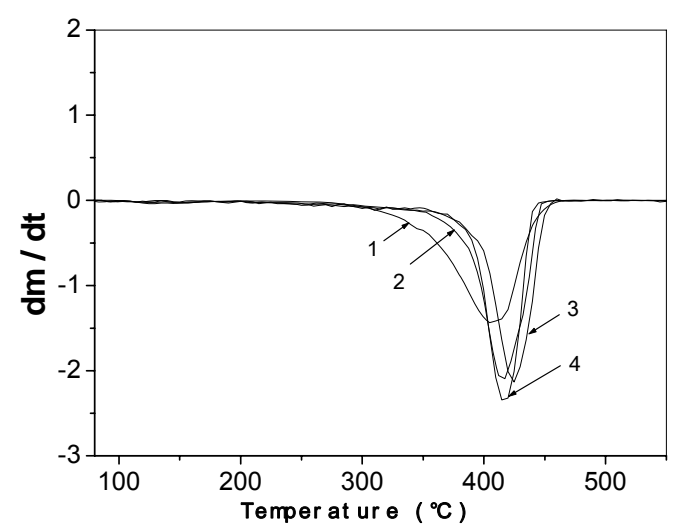

(b)

Fig. 6. The TG (a) and DTG (b) curves of polymer/OMMT nanocomposites with DVB content of $2 \mathrm{wt} \%$ and various contents of OMMT: 0 (1), 3 (2), 5 (3) and $8 w t \%$ (4).

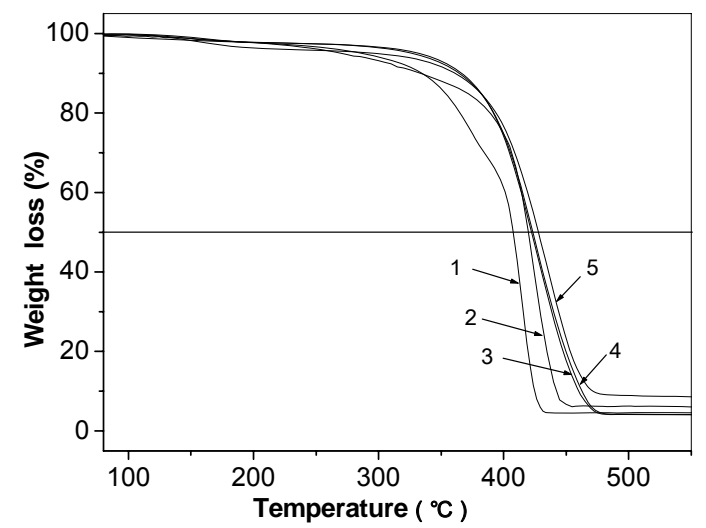

(a)

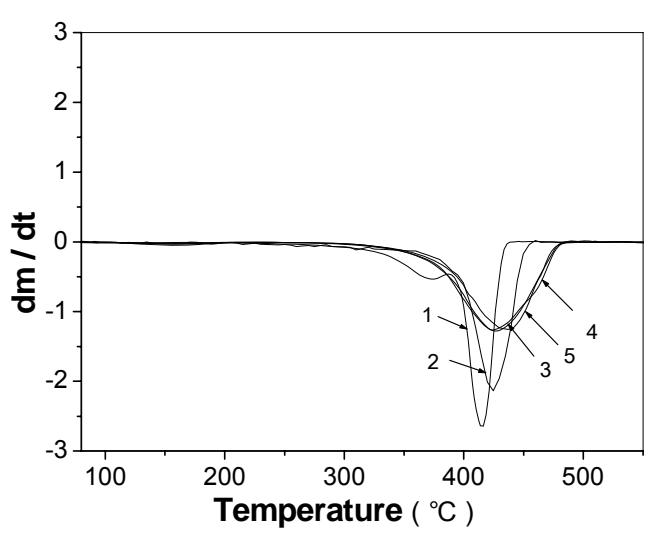

(b)

Fig. 7. The TG (a) and DTG (b) curves of polymer/OMMT nanocomposites with OMMT content of $5 \mathrm{wt} \%$ and various contents of DVB: 0 (1), 2 (2), 4 (3), 6 (4) and $10 \mathrm{wt} \%(5)$.

\section{Microstructure of polymer/clay nanocomposites}

Fig. 8 shows the TEM images of the polymer/OMMT nanocomposites prepared with OMMT content of 3,5 and $8 \mathrm{wt} \%$ by suspension polymerization. The dispersed layer of montmorillonite (dark lines), alternate with the chains of the polymer (white part), 
can be observed. With increasing the content of the OMMT (Fig.8a, Fig.8b and Fig.8c), the OMMT layers assemble to an agglomeration, which clearly show that the dispersion of OMMT was better at a lower content than at a higher content. It is believed that the formation of agglomeration in polymer/OMMT nanocomposites is due to the hydroxylated edge-edge interaction of the silicate layers [22].
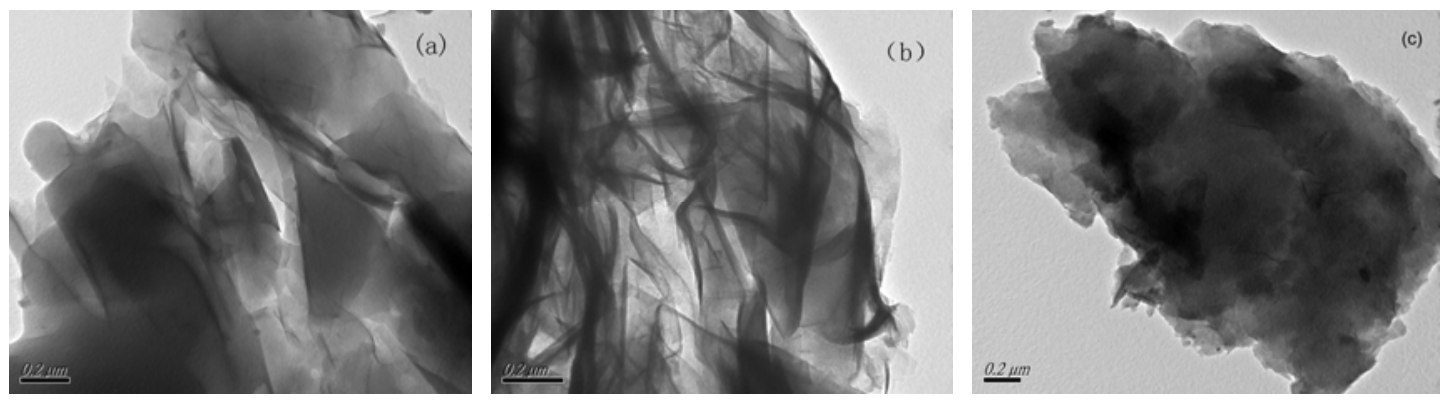

Fig. 8. TEM micrographs of polymer/OMMT nanocomposites with OMMT content of 3 wt\% (a), 5 wt\% (b) and 8 wt\% (c).
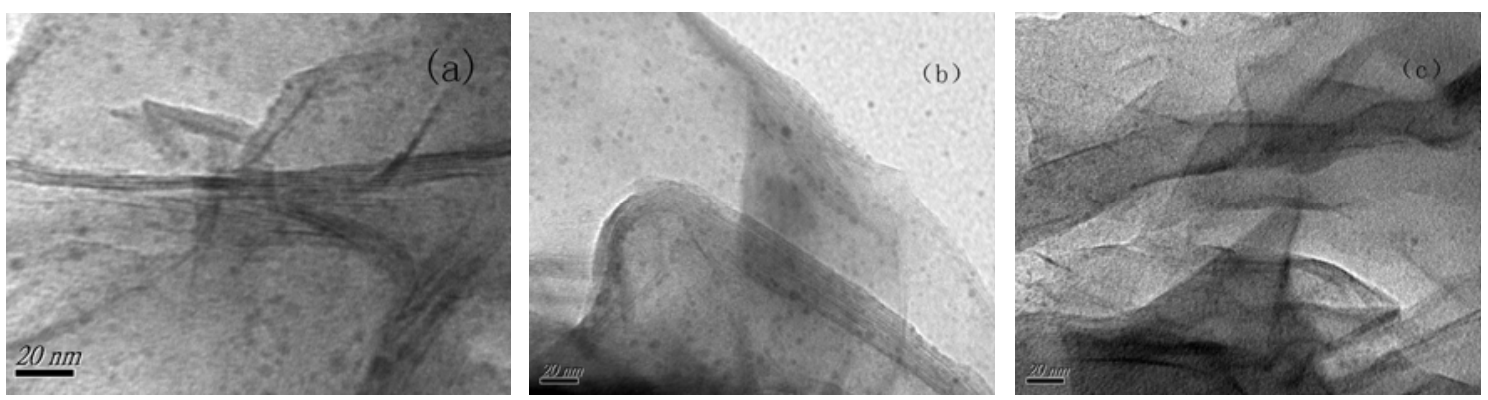

Fig. 9. TEM micrographs of polymer/OMMT nanocomposites with OMMT content of 5 wt\%.

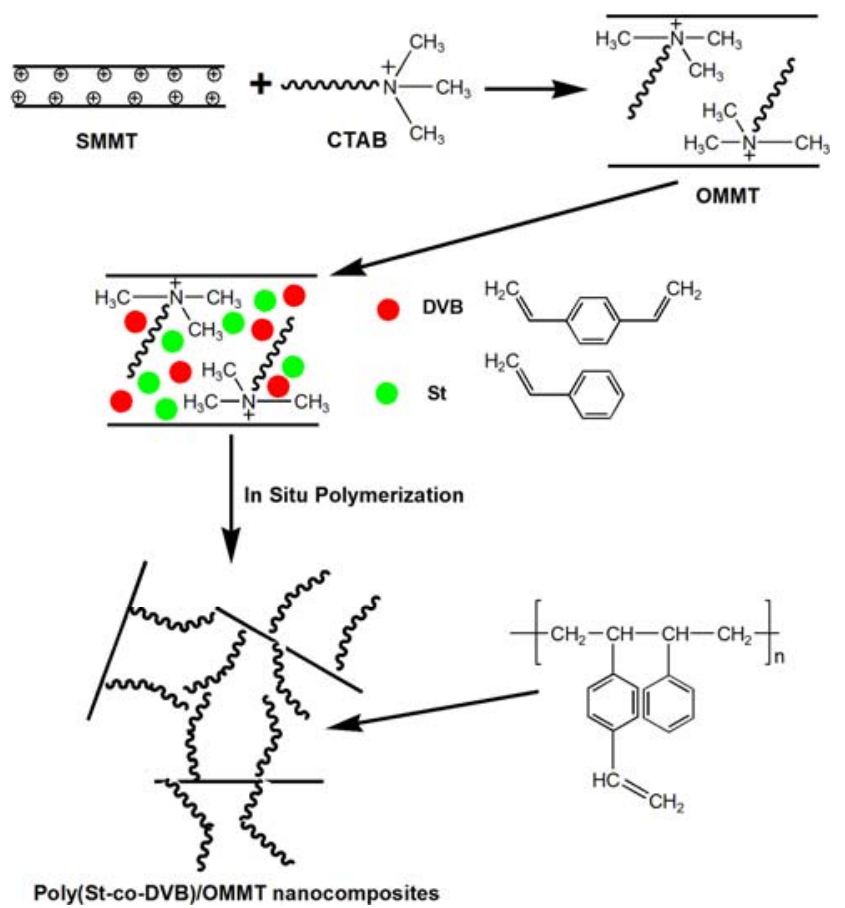

Fig. 10. The model of intercalation polymerization. 
Fig. 9 shows the TEM images of the polymer/OMMT nanocomposites with OMMT content of $5 \mathrm{wt} \%$ prepared by suspension polymerization. It can be seen from the figure that the chains of the polymer had been partly intercalated into the layers of the montmorillonite and the polymer/OMMT layered structure nanocomposite has formed. This result has been confirmed by XRD (Fig. 4 and Fig. 5). The intercalation model is shown in Fig.10.
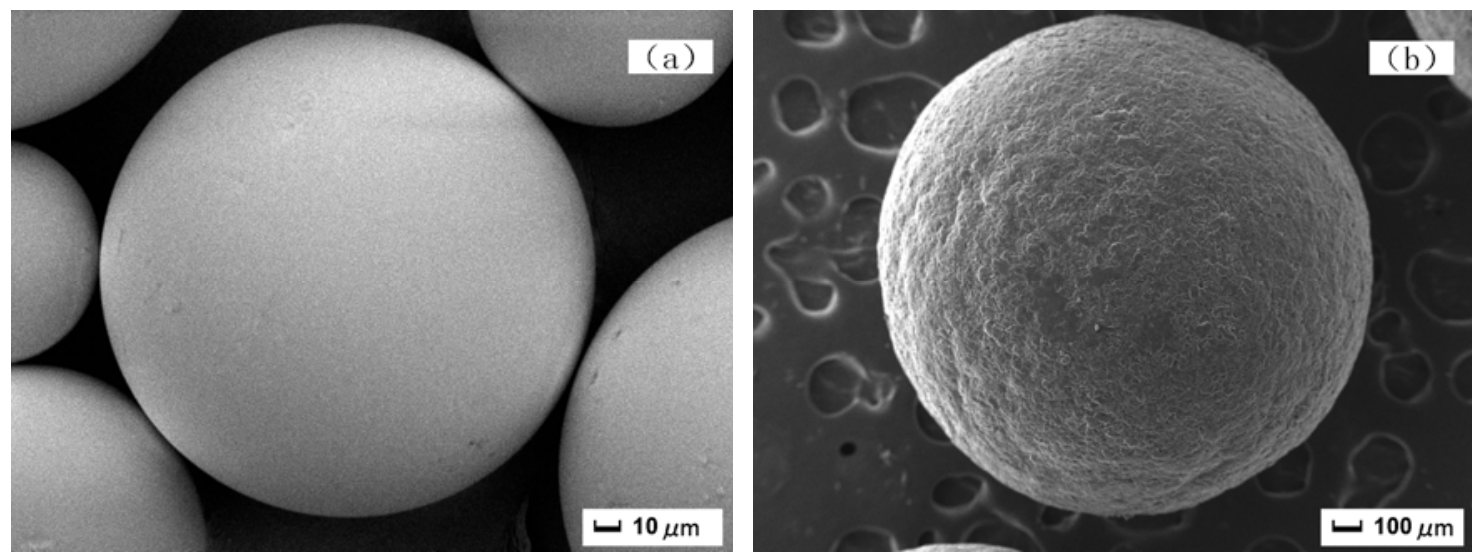

Fig. 11. SEM micrograph of pure polymer (a) and polymer/OMMT nanocomposites (b) with OMMT content of $5 \mathrm{wt} \%$.

Fig. 11. shows SEM micrographs of pure polymer and polymer/OMMT nanocomposite with OMMT content of $5 \mathrm{wt} \%$. Both pure polymer and polymer/OMMT nanocomposite exhibited spherical shape, whereas the polymer/OMMT nanocomposite lost the smooth surfaced of polymer and a lot of pits were formed in the spherical surface. In the present system, there were a few monomers and oligomers assemble at surface of microspheres and the monomers and oligomers were washed out by ethanol. This could probably be due to the fact that montmorillonite acts as a inhibitor that has a chain transfer termination effect in the formation of the polymer chain and restrict the polymerization of the polymer chains. This lead to the molecular weight decline [23].

\section{Conclusions}

The polymer/OMMT nanocomposites were synthesized by suspension polymerization. The XRD and TEM results showed that the polymer chains had inserted into the interlayer of clay and the maximum interlayer spacing of nanocomposite was $4.0 \mathrm{~nm}$. The TG and DTG results indicated that the cross-linked polymer/clay nanocomposites exhibited better thermal stability than pure polystyrene and polystyrene/clay nanocomposites. When the OMMT content is $5 \mathrm{wt} \%$, the nanocomposites had the best thermal stability under the experimental conditions.

\section{Experimental part}

\section{Materials}

Sodium montmorillonite was offered by Zhejiang Fenghong Clay Chemicals CO., LTD (China). Divinylbenzene (DVB) (industrial $55 \mathrm{wt} \%$ ) was obtained from Danyang Chemical Reagent Co., LTD (China). Styrene, cetyltrimethyl ammonium bromide (CTAB), polyvinyl alcohol (PVA), and benzyl peroxide (BPO) were purchased from Shanghai Chemical Reagent Co., LTD (China). All reagents were used without further 
purification.

\section{Preparation of organic montmorillonite}

Organic montmorillonite (OMMT) was prepared by ion exchange method between sodium montmorillonite (SMMT) and cetyltrimethyl ammonium bromide (CTAB). The suspension solution contained $50 \mathrm{~g}$ of SMMT, $13 \mathrm{~g}$ of CTAB and $650 \mathrm{ml}$ of distilled water, and then stirred at $75{ }^{\circ} \mathrm{C}$ with a ultrasonic mixer for $4 \mathrm{~h}$. The products were filtered and washed with distilled water until no bromine ion was detected, then dried in vacuum oven at $60{ }^{\circ} \mathrm{C}$ for $12 \mathrm{~h}$.

\section{Preparation of polymer/clay nanocomposites by suspension polymerization}

Poly(St-co-DVB)/OMMT nanocomposites were prepared by suspension polymerization. $0.2 \mathrm{~g}$ of the dispersion agent (PVA) was dissolved in $200 \mathrm{ml}$ of distilled water at $80{ }^{\circ} \mathrm{C}$. The initiator benzyl peroxide (BPO) was added to the mixed solution of styrene and divinylbenzene (DVB). The molar ratio of BPO to monomer was 1:200. The various amount of OMMT was dispersed in the mixed solution of styrene and DVB at room temperature. The experimental conditions are shown in Table 1. Then, the mixed solution was added to the PVA solution and stirred maintaining at $80{ }^{\circ} \mathrm{C}$. After $8 \mathrm{~h}$ polymerization, the products were filtered and washed with ethanol, then dried in vacuum oven at $60{ }^{\circ} \mathrm{C}$ for $12 \mathrm{~h}$.

Tab. 1. The experimental conditions of polymer/OMMT nanocomposites.

\begin{tabular}{|c|c|c|c|c|c|c|c|}
\hline No & DVB & St & OMMT (\%) & No & DVB & St & OMMT (\%) \\
\hline 1 & & 100 & 0 & 11 & 4 & 96 & 5 \\
\hline 2 & 0 & 100 & 3 & 12 & & 96 & 8 \\
\hline 3 & & 100 & 5 & 13 & & 94 & 0 \\
\hline 4 & & 100 & 8 & 14 & 6 & 94 & 3 \\
\hline 5 & & 98 & 0 & 15 & & 94 & 5 \\
\hline 6 & 2 & 98 & 3 & 16 & & 94 & 8 \\
\hline 7 & & 98 & 5 & 17 & & 90 & 0 \\
\hline 8 & & 98 & 8 & 18 & 10 & 90 & 3 \\
\hline 9 & 4 & 96 & 0 & 19 & & 90 & 5 \\
\hline 10 & & 96 & 3 & 20 & & 90 & 8 \\
\hline
\end{tabular}

\section{Instruments and characterization}

Fourier transform infrared (FT-IR) absorption spectra were obtained with FT-IR spectrometer Nicolet NEXUS 470 in the range of $4000-400 \mathrm{~cm}^{-1}$. X-ray diffraction patterns were obtained by using D8 super speed X-ray diffractometer equipped with $\mathrm{Cu} \mathrm{Ka}$ radiation $(\lambda=0.514 \mathrm{~nm})$. The scanning range was $1-10^{\circ}$. The thermal gravimetric analysis was conducted on NETZSCH STA449C under $\mathrm{N}_{2}$ flow from $25{ }^{\circ} \mathrm{C}$ to $550{ }^{\circ} \mathrm{C}$ with heating rate $10{ }^{\circ} \mathrm{C} / \mathrm{min}$. The surface morphology and microstructure were investigated by JSM-7001F scanning electron microscope (SEM) and JEM-2100 transmission electron microscope (TEM), respectively. 


\section{Acknowledgements}

The authors acknowledge financial support by the National High Technology Research and Development Program of China (No. 2007AA03Z358).

\section{References}

[1] Sur, G.S.; Sun, H.L. et al. Polymer , 2001; 42, 9783.

[2] Messersmith, P.B.; Giannelis, E.P. J Polym Sci Polym Chem,1995, 33:1047.

[3] Lim, S.T.; Lee, C.H. et al. e-polymers , 2004, 026.

[4] Morlat, S.; Mailhot, B. et al. Chem Mater, 2004, 16:377.

[5] Beyer, G. Plast Addit Compound 2002; 4: 22.

[6] Kannan, M.; Bhagawan, S.S. et al. e-polymers 2008; no. 133.

[7] Lee, S.R., Park, H.M. et al. Polymer, 2002; 43: 2495.

[8] Miroslava, M.; Kada, B. et al. e-polymers, 2005, 019.

[9] Gefu, J.; Guoqiang, L.. Mater Sci Eng A 2008; $498: 327$.

[10] Kim, H.B.; Hyun, Y.H. et al. Mater Sci Eng C 2006; 26: 306.

[11] Praus, P. et al. J Colloid Interface Sci 2006, 304, 29.

[12] Huskic, M. et al. J Non-Cryst Solids, 2008, 354: 3326.

[13] Rodríguez, Y.et al. Appl Clay Sci 2009; 43:91.

[14] Tabtiang, A. et al. Euro Polym J 2000, 36:137.

[15] Yang, K. et al. Polymer, 2007, 48:6254.

[16] Yang, W.T.; Ko, T.H. et al. Polymer Composites 2008; 10: 409.

[17] Yoon, S.K.; Byun, B.S. et al. Journal of Industrial and Engineering Chemistry 2008; $14: 417$.

[18] Sivudu, S.K.; Thomas, S.; Shailaja, D. Applied Clay Science 2007; 37: 185.

[19] Changa, J-H.; Kim, S.J. et al.Polymer 2004, 45, 919.

[20] Morgan, A.B.; Gilman, J.W. Journal of Applied Polymer Science 2003, 87, 788.

[21] Miwa, Y.; Andrew, R. et.al. Macromolecules 2008; 41(13):4701.

[22] Ray, S.S.; Okamoto, K. et al. Macromolecules, 2003, 36, 2355.

[23] Hwu, J.M.; Ko, T.H. et al. Journal of Applied Polymer Science 2004; 91: 101. 